\title{
Impacto de fatores sociodemográficos e comportamentais na prevalência de sobrepeso e obesidade de escolares
}

\author{
Impact of sociodemographic and behavioral factors on the \\ prevalence of overweight and obesity in schoolchildren
}

\author{
Dartagnan Pinto Guedes \\ Jaime Tolentino Miranda Neto \\ Maria João Almeida \\ António José Rocha Martins e Silva ${ }^{3}$
}

1 Universidade Estadual de Londrina, Paraná, Brasil

2 Universidade Estadual de Montes Claros, Minas Gerais, Brasil

3 Centro de Investigação em Desporto, Saúde e De senvolvimento Humano, Universidade de Trás-osMontes e Alto Douro, Vila Real, Portugal

Recebido em 13/10/09 Revisado em 21/12/09 Aprovado em 21/01/10
Resumo - O estudo analisou o impacto de fatores sociodemográficos e comportamentais na prevalência de sobrepeso e obesidade em amostra representativa de escolares de Montes Claros, Minas Gerais, Brasil. A amostra foi constituída por 2849 sujeitos (1457 moças e 1392 rapazes), com idades entre 6 e 18 anos. Sobrepeso e obesidade foram definidos mediante o índice de massa corporal, adotando-se pontos-de-corte para gênero e idade sugeridos pela International Obesity Task Force (IOFT). As prevalências nos diferentes estratos associados aos fatores sociodemográficos e comportamentais foram comparadas por intermédio do cálculo de regressão logística, utilizando-se odds ratios ajustadas. Entre as moças, as prevalências de sobrepeso e obesidade encontradas foram de $19,7 \%$ e 4,8\%, respectivamente, enquanto entre os rapazes, $14,7 \%$ e $2,8 \%$. Os resultados encontrados mostram que, embora as prevalências do sobrepeso e da obesidade sejam comparáveis com estimativas observadas em outras regiões brasileiras e menores que as encontradas em regiões mais industrializadas, a proporção de escolares que apresentaram sobrepeso e são obesos é particularmente preocupante. Os principais fatores sociodemográficos e comportamentais identificados na ocorrência do sobrepeso e da obesidade foram classe econômica, escolaridade dos pais, número de irmãos, tipo de alimentação na escola, distância e meio de transporte entre a casa e a escola, realização de trabalho remunerado e tipo de atividade no lazer e no tempo livre. Os dados mostram que existe urgente necessidade de implementar programas de in tervenção educacional direcionados à promoção da prática de atividade física e de hábitos dietéticos adequados.

Palavras-chave: Índice de massa corporal; IOTF; Fatores de risco; Crianças; Adolescentes.

Abstract - This study analyzed the impact of sociodemographic and behavioral factors on the prevalence of overweight and obesity in a representative sample of schoolchildren from Montes Claros, Minas Gerais, Brazil. The sample consisted of 2849 children and adolescents (1457 girls and 1392 boys), ranging in age from 6 to 18 years. Overweight and obesity were defined based on body mass index according to the cut-off values for gender and age suggested by the International Obesity Task Force (IOTF). The prevalence rates in the different strata associated with sociodemographic and behavioral factors were compared by logistic regression using adjusted odds ratios. The prevalence of overweight and obesity was $19.7 \%$ and $4.8 \%$, respectively, among girls, and $14.7 \%$ and $2.8 \%$ among boys. The results show that, although the prevalence of overweight and obesity is similar to estimates observed in other Brazilian regions and smaller than those found in more industrialized areas, the proportion of schoolchildren who presented overweight and obesity is a matter of concern. The main sociodemographic and behavioral factors associated with the occurrence of overweight were economic status, educational level of the parents, number of siblings, school menu, distance and means of transportation between home and school, paid work, and leisure-time activity. The data show an urgent need for the implementation of educational intervention programs designed to promote physical activity and adequate dietary habits.

Key words: Body mass index; IOTF; Risk factors; Children; Adolescents. 


\section{INTRODUÇÃO}

Estudos realizados em diferentes regiões do mundo têm apontado acentuado aumento na prevalência de sobrepeso e obesidade em populações jovens ${ }^{1}$. Especificamente no Brasil, no início da década de 1970, a prevalência de sobrepeso em jovens entre 6 e 18 anos era estimada em 4\%. Na década de 1990, essa estimativa se elevou para 14\%, e em 2003 alcançou proporção próxima de $17 \%$, ou seja, mais que quadruplicou ${ }^{2}$.

Estes achados preocupam sobremaneira aqueles que limitam na área de saúde pública, considerando que a associação entre sobrepeso/obesidade e alterações metabólicas, como é o caso das dislipidemias, intolerância à glicose e hipertensão, apontados como fatores de risco predisponentes às doenças cardiovasculares e ao diabetes melitus tipo 2, que até pouco tempo atrás era mais evidente em adultos, já pode ser identificadas com freqüência na infância e na adolescência ${ }^{3}$.

Além das implicações biológicas para a saúde dos jovens, crianças e adolescentes com sobrepeso e obesos apresentam maior incidência de problemas relacionados à auto-estima e ao auto-conceito, afetando o relacionamento interpessoal com graves repercussões psicológicas e sociais ${ }^{4}$.

O sobrepeso e a obesidade apresentam etiologia multicausal, envolvendo componentes genéticos, fisiológicos, metabólicos e psicológicos. Contudo, o que pode justificar em grande parte o crescente aumento nas últimas décadas da proporção de jovens com sobrepeso e obesos são as mudanças observadas recentemente em seu estilo de vida. Dessa forma, tentar compreender possíveis indicadores ambientais associados à prevalência de sobrepeso e obesidade de população específica poderá oferecer substanciais contribuições para os programas de intervenção.

Sendo assim, o objetivo do estudo foi analisar o impacto de selecionados fatores sociodemográficos e comportamentais na prevalência de sobrepeso e obesidade da população escolar de Montes Claros, Minas Gerais, Brasil.

\section{PROCEDIMENTOS METODOLÓGICOS}

Para elaboração do estudo foram utilizadas informações contidas em banco de dados construído a partir de levantamento descritivo de corte transversal de base populacional envolvendo escolares do município de Montes Claros, Minas Gerais, Brasil. A coleta dos dados foi realizada de abril a novembro de 2007 e os protocolos de intervenção utilizados foram aprovados pelo Comitê de Ética em Pesquisa da Universidade Estadual de Montes Claros - UNIMONTES (processo 529/2006) e acompanharam normas da Resolução 196/96 do Conselho Nacional de Saúde sobre pesquisa envolvendo seres humanos.

A cidade de Montes Claros, cenário do presente estudo, localiza-se no norte do estado de Minas Gerais, Brasil. Para sua caracterização, assumindo como referência o Índice de Desenvolvimento Humano (IDH), verifica-se que a cidade de Montes Claros, nas duas últimas décadas, vem apresentando importante evolução. Contudo, ainda assim, apresenta valores equivalentes ao IDH menores em comparação com cidades de similar densidade populacional localizadas em outras regiões do Brasil, sobretudo, da região sudeste e sul. Como ilustração, no período de 1991-2006, o IDH de Montes Claros cresceu 10\%, passando de 0,713 em 1991 para 0,783 em 2006. A dimensão que mais contribuiu para este crescimento foi educação $(0,793$ versus 0,872$)$ seguida da renda $(0,629$ versus 0,681$)$ e da longevidade $(0,741$ versus 0,787$)$. Neste período, o hiato de desenvolvimento humano, ou seja, a distância entre o IDH do município e o limite máximo de IDH (1 - IDH), foi reduzido em 32\%. O IDH-2006 médio das cidades localizadas nas regiões sudeste e sul é 0,844 e 0,825, respectivamente, e o IDH-2006 brasileiro $0,800^{5}$.

\section{Amostra e seleção dos sujeitos}

A população de referência para o estudo incluiu os escolares de ambos os gêneros, entre 6 e 18 anos de idade, que estavam regularmente matriculados no ano letivo 2007, nas escolas de ensino fundamental e médio das redes de ensino público (estadual e municipal) e privado do município de Montes Claros, Minas Gerais, Brasil. Para ilustrar a dimensão do universo populacional tratado, de acordo com informações do Setor de Estatística da Secretaria da Educação do Estado de Minas Gerais, estavam matriculados no início do ano letivo de 2007, nas 237 escolas de ensino fundamental e médio localizadas na região urbana do município, 84.675 escolares, sendo 45.033 do gênero feminino e 39.642 do gênero masculino.

O tamanho da amostra foi estabelecido assumindo intervalo de confiança de $95 \%$, erro amostral de $3 \%$ e acréscimo de $10 \%$ para atender eventuais casos de perdas na coleta dos dados. Considerando que o planejamento amostral envolveu conglomerados, definiu-se efeito do delineamento da amostra (deff) equivalente a 1,5, prevendo 
inicialmente amostra mínima de 2600 escolares. Porém, a amostra definitiva utilizada no tratamento das informações foi composta por 2849 escolares (1457 moças e 1392 rapazes).

Quanto à seleção dos escolares, procurou-se obter amostragem probabilística por conglomerados, tendo como referência o número de escolares quanto ao gênero, à série e ao turno em que estavam matriculados em cada estrato da estrutura escolar separadamente (estadual, municipal e privada). Os critérios adotados para a exclusão de algum escolar sorteado para o estudo foram: (a) recusa em participar do estudo; (b) não-autorização dos pais ou responsáveis; (c) ausência às aulas no dia agendado para a coleta dos dados; (d) algum problema físico que o impedisse, temporária ou definitivamente, de realizar as medidas antropométricas.

\section{Coleta dos dados}

A idade cronológica dos escolares foi determinada de forma centesimal, a partir da confrontação entre a data de coleta dos dados e a data de nascimento. No entanto, para feito de análise dos dados, optou-se pela constituição de três grupos etários. O primeiro grupo etário reuniu escolares de 6 a 9 anos; o segundo grupo etário de 10 a 14 anos; e o terceiro grupo etário de 15 a 18 anos de idade.

Quanto aos indicadores sociodemográficos e comportamentais foram coletadas informações quanto à cor da pele, à classe econômica familiar, à escolaridade dos pais, à agregação familiar, à quantidade de irmãos, ao turno que estuda, ao número de repetências, à realização de refeições na escola, à distância entre a residência e a escola, ao meio de transporte para se deslocar da residência para a escola, à realização de trabalho remunerado e ao tipo de atividade no lazer e no tempo livre. Para classificação econômica das famílias dos escolares, recorreram-se as diretrizes propostas pela Associação Brasileira das Empresas de Pesquisa, com base no nível de escolaridade do chefe da família, condições de moradia, posse de utensílios domésticos, automóveis e número de empregados domésticos ${ }^{6}$. Mediante pontuações computadas em cada um dos itens considerados foi definida a classe econômica familiar categorizada do maior nível (classe A) para o menor nível (classe E).

O sobrepeso e a obesidade foram definidos a partir do cálculo do índice de massa corporal (IMC), razão entre as medidas de massa corporal expressa em quilogramas e da estatura expressa em metros ao quadrado $\left(\mathrm{kg} / \mathrm{m}^{2}\right)$, adotando-se os pontos-de-corte para gênero e idade sugeridos no estudo promovido pela International Obesity Task Force - IOTF? . Para a determinação das medidas equivalentes à massa corporal foi empregada balança antropométrica com definição de 10 gramas, aferida a cada dez pesagens, enquanto para a realização das medidas de estatura utilizou-se estadiômetro de alumínio com escala de $1 \mathrm{~mm}$, a partir dos procedimentos apresentados pela Organização Mundial da Saúde 8 .

\section{Tratamento estatístico}

O tratamento estatístico dos dados foi realizado mediante pacote computadorizado Statistical Package for the Social Science (SPSS), versão 17.0. Os dados antropométricos foram inicialmente comparados com a curva normal utilizando o teste de distância K-S (Kolmogorov-Smirnov). As medidas de massa corporal, estatura e IMC mostraram distribuição de freqüência normal.

Para estabelecer as prevalências de sobrepeso e obesidade recorreram-se as proporções de freqüência e respectivos intervalos de confiança de 95\%, de acordo com pontos-de-corte empregados. Para verificar eventuais diferenças entre as prevalências de sobrepeso e obesidade em cada estrato considerado utilizou-se do teste de significância para comparações de múltiplas proporções, mediante o envolvimento da estatística de qui-quadrado $\left(\chi^{2}\right)$.

Para analisar o impacto dos fatores sociodemográficos e comportamentais na prevalência de sobrepeso dos escolares recorreram-se aos cálculos dos valores de odds ratio (OR), estabelecidos por intermédio da regressão logística binária, mediante análise bruta e ajustada pelas demais variáveis independentes envolvidas nos modelos de regressão. Para modelagem da regressão logística foram consideradas apenas aquelas variáveis que apresentaram significância $\leq 0,20$ para associação com a prevalência de sobrepeso no teste $\chi^{2}$.

\section{RESULTADOS}

A tabela 1 mostra as prevalências de sobrepeso e obesidade estimadas na população escolar analisada de acordo com gênero e grupo etário. Ao levar em conta o conjunto das idades consideradas, a prevalência de sobrepeso foi equivalente a 19,7\% entre as moças e $14,7 \%$ entre os rapazes, dos quais, $4,8 \%$ e 2,8\%, respectivamente, demonstraram ser obesos. Com relação aos grupos etários considerados, em ambos os gêneros, verificam-se aumentos significativos nas prevalências de sobrepeso e obesidade com a idade. A prevalência de sobrepeso iniciou 
Tabela 1. Prevalência (95\% IC) de sobrepeso e obesidade de acordo com gênero e grupo etário na população escolar de Montes Claros, Minas Gerais, Brasil, 2007.

\begin{tabular}{lccccc}
\hline Grupo Etário & Sobrepeso & Obesidade & Moças & Rapazes \\
\hline$\leq 9$ Anos & $13.7(12.2-15.3)$ & $8.7(8.2-9.2)$ & $3.2(2.8-3.6)$ & $1.9(1.7-2.2)$ & $1.9(1.7-2.2)$ \\
$10-14$ Anos & $17.1(15.4-18.9)$ & $13.4(11.7-15.2)$ & $4.7(4.2-5.3)$ & $2.6(2.1-3.1)$ & $2.6(2.1-3.1)$ \\
$\geq 15$ Anos & $28.4(26.3-30.7)$ & $21.9(19.8-24.1)$ & $6.4(5.7-7.2)$ & $4.0(3.3-4.8)$ & $4.0(3.3-4.8)$ \\
$6-18$ Anos & $19.7(17.9-21.8)$ & $14.7(13.1-16.5)$ & $4.8(4.2-5.5)$ & $2.8(2.4-3.3$ & $2.8(2.4-3.3)$ \\
\hline
\end{tabular}

nos escolares $\leq 9$ anos com proporções de 13,7\% nas moças e $8,7 \%$ nos rapazes, e alcançou proporções de $28,4 \%$ e $21,9 \%$, respectivamente, nos escolares $\geq$ 15 anos. De maneira similar, porém em proporções menores, a prevalência de obesidade nos escolares de ambos os gêneros com idades $\geq 15$ anos foi duas vezes maior (6,4\% nas moças e 4,0\% nos rapazes) quando comparados com seus pares com idades $\leq$ 9 anos (3,2\% e 1,9\%, respectivamente).

As análises das prevalências de sobrepeso e obesidade com estratificação para cada fator sociodemográfico e comportamental selecionado para o estudo podem ser observadas na tabela 2. Mediante o teste $\chi^{2}$ é constatado que as diferenças proporcionais observadas na ocorrência do sobrepeso e da obesidade estratificada pela cor de pele, agregação familiar, turno que estuda e número de repetências não são apontadas estatisticamente $(\mathrm{p}<0,20)$.

A tabela 3 procura apresentar o impacto dos fatores sociodemográficos e comportamentais que se mostraram significativamente associadas às prevalências de sobrepeso observadas na população escolar analisada. Mediante análise dos resultados da regressão logística, mesmo considerando ajuste por todas as demais variáveis independentes envolvidas no modelo de regressão, estima-se que as moças escolares de Montes Claros, Minas Gerais, evidenciam risco $41 \%$ maior de apresentarem sobrepeso que os rapazes $(\mathrm{OR}=1,41 ; 95 \%$ IC 1,04-1,80). Com relação aos grupos etários considerados, verificam-se tendências de aumento nas prevalências de sobrepeso com a idade. $\mathrm{O}$ risco dos escolares analisados no estudo com idades $\geq 15$ anos de apresentarem sobrepeso é por volta de duas vezes e meia maior quando comparado com seus pares $\leq$ 9 anos (OR = 2,46; 95\% IC 2,04-2,91), independentemente da participação simultânea de qualquer outro fator envolvido no modelo estatístico.

Quanto às classes econômicas familiar, as estimativas encontradas revelam que as prevalências associadas ao sobrepeso apresentaram valores progressivamente maiores de acordo com posições econômicas mais elevadas. Aqueles escolares pertencentes as famílias de classe econômica mais privilegiada (classe A) demonstraram possuir risco, em valores aproximados, mais que o dobro maior de apresentarem sobrepeso (OR $=2,23 ; 95 \%$ IC 1,82-2,66) que escolares pertencentes às famílias de classe econômica mais baixa (classe E). Contudo, quando considerados os valores ajustados pelas demais variáveis independentes do modelo de regressão, o impacto das classes econômicas nas prevalências do sobrepeso diminui, no entanto, permanece significativo $(\mathrm{OR}=1,57 ; 95 \%$ IC $1,28-1,91)$.

A escolaridade do pai ou do responsável pelo sustento do escolar e o número de irmãos são outros dois fatores vinculados ao contexto familiar que apresentam algum impacto na ocorrência do sobrepeso. Aqueles escolares em que o pai ou o responsável alcançou $\geq 12$ anos de escolarização, controlando as demais variáveis independentes envolvidas no modelo de regressão, possuem uma vez e meia mais chance de apresentar sobrepeso $(\mathrm{OR}=1,59 ; 95 \%$ IC $1,34-1,88)$ quando comparados aos seus pares em que o pai ou o responsável não completou mais que quatro anos de escolarização. Comparativamente com aqueles em que o pai ou o responsável tem menor escolarização, as dimensões das OR apontam que os riscos dos escolares apresentarem sobrepeso tornam-se significativos a partir do nono ano de escolarização do pai ou do responsável.

Ao considerar o número de irmãos, constatase que as prevalências equivalentes ao sobrepeso diminuem de acordo com o aumento no número de irmãos apresentado pelos escolares. Aqueles que relataram ser filho único demonstram por volta de $81 \%$ mais chance de apresentar sobrepeso (OR $=1,81 ; 95 \%$ IC 1,37 - 2,27) quando comparados aos que relataram possuir cinco ou mais irmãos. Contudo, ao estabelecer controle estatístico das demais variáveis independentes consideradas no estudo, as chances dos escolares que não têm irmãos apresentarem sobrepeso se reduz para $42 \%(\mathrm{OR}=$ 1,42; 95\% IC 1,09 - 1,80). Ainda, apesar da relação inversa entre o número de irmãos e a magnitude 
Tabela 2. Prevalência (95\% IC) de sobrepeso e obesidade de acordo com selecionadas variáveis sociodemográficas e comportamentais da população escolar de Montes Claros, Minas Gerais, Brasil, 2007.

\begin{tabular}{|c|c|c|c|c|}
\hline & \multicolumn{2}{|c|}{ Sobrepeso } & \multicolumn{2}{|c|}{ Obesidade } \\
\hline \multicolumn{5}{|l|}{ Gênero } \\
\hline Moças & $19.7(17.9-21.8)$ & \multirow{2}{*}{0.109} & $4.8(4.3-5.5)$ & \multirow{2}{*}{0.057} \\
\hline Rapazes & $14.7(13.1-16.5)$ & & $2.8(2.4-3.3)$ & \\
\hline$\leq 9$ Anos & $11.2(10.0-12.6)$ & \multirow{3}{*}{0.000} & $2.6(2.3-3.0)$ & \multirow{3}{*}{0.001} \\
\hline $10-14$ Anos & $15.3(13.9-16.8)$ & & $3.7(3.2-4.3)$ & \\
\hline$\geq 15$ Anos & $25.2(23.4-27.1)$ & & $5.2(4.4-6.1)$ & \\
\hline \multicolumn{4}{|l|}{ Cor da Pele } & 0.327 \\
\hline Classe E & $10.2(9.3-11.1)$ & \multirow{5}{*}{0.026} & $2.5(2.1-3.0)$ & \multirow{5}{*}{0.014} \\
\hline Classe D & $10.5(9.6-11.4)$ & & $2.6(2.2-3.1)$ & \\
\hline Classe C & $14.6(13.3-16.0)$ & & $3.5(3.0-4.0)$ & \\
\hline Classe B & $21.4(19.8-23.1)$ & & $5.0(4.4-5.6)$ & \\
\hline Classe A & $22.3(20.7-24.0)$ & & $5.2(4.5-6.0)$ & \\
\hline \multicolumn{5}{|c|}{ Escolaridade dos Pais } \\
\hline$\leq 4$ Anos & $11.6(10.4-12.9)$ & 0.049 & $2.8(2.4-3.3)$ & 0.036 \\
\hline Pai e Mãe & $17.0(15.8-18.2)$ & \multirow[t]{2}{*}{0.497} & $3.6(3.2-4.1)$ & \multirow[t]{2}{*}{0.563} \\
\hline Pais Separados & $17.9(16.5-19.4)$ & & $3.9(3.4-4.5)$ & \\
\hline \multicolumn{5}{|l|}{ Número de Irmãos } \\
\hline$\geq 5$ & $10.5(9.6-11.5)$ & \multirow{4}{*}{0.031} & $2.3(1.9-2.8)$ & \multirow{4}{*}{0.000} \\
\hline $3-4$ & $12.9(11.6-14.3)$ & & $3.3(2.9-3.7)$ & \\
\hline $1-2$ & $16.3(14.9-17.8)$ & & $3.8(3.4-4.2)$ & \\
\hline Nenhum & $23.8(22.2-25.6)$ & & $5.6(5.0-6.3)$ & \\
\hline \multicolumn{4}{|l|}{ Turno de Estudo } & \multirow{3}{*}{0.476} \\
\hline Manhã & $16.6(15.1-18.2)$ & 0.527 & $3.6(3.1-4.1)$ & \\
\hline Tarde & $17.8(16.4-19.3)$ & & $3.9(3.4-4.4)$ & \\
\hline \multicolumn{5}{|l|}{ Repetência } \\
\hline$\geq 3$ & $16.2(14.9-17.7)$ & & $3.5(3.1-4.0)$ & \\
\hline $1-2$ & $16.9(15.5-18.4)$ & 0.235 & $3.7(3.3-4.2)$ & 0.348 \\
\hline Nenhuma & $18.4(16.8-20.1)$ & & $4.0(3.5-4.6)$ & \\
\hline
\end{tabular}




\begin{tabular}{|c|c|c|c|c|}
\hline & \multicolumn{2}{|c|}{ Sobrepeso } & \multicolumn{2}{|c|}{ Obesidade } \\
\hline & Prevalência (95\% IC) & $\mathrm{p}$ & Prevalência (95\% IC) & $\mathrm{p}$ \\
\hline \multicolumn{5}{|c|}{ Distância Casa-Escola } \\
\hline$>10 \mathrm{~km}$ & $11.9(10.6-13.3)$ & \multirow{4}{*}{0.036} & $2.9(2.5-3.4)$ & \multirow{4}{*}{0.041} \\
\hline $5-10 \mathrm{~km}$ & $13.8(12.4-15.3)$ & & $3.4(3.0-3.8)$ & \\
\hline $1-5 \mathrm{~km}$ & $18.3(16.8-19.9)$ & & $4.3(3.8-4.9)$ & \\
\hline$<1 \mathrm{~km}$ & $19.5(17.9-21.3)$ & & $4.6(4.1-5.2)$ & \\
\hline \multicolumn{4}{|c|}{ Transporte para Escola } & \multirow{4}{*}{0.036} \\
\hline Caminhada/Bicicleta & $10.8(9.4-12.4)$ & \multirow{3}{*}{0.045} & $2.9(2.5-3.4)$ & \\
\hline Transporte Coletivo & $16.3(14.8-17.9)$ & & $3.9(3.4-4.5)$ & \\
\hline Carro & $20.2(18.7-21.9)$ & & $4.7(4.1-5.4)$ & \\
\hline \multicolumn{5}{|c|}{ Trabalho Remunerado } \\
\hline Não & $13.7(12.3-15.2)$ & \multirow{4}{*}{0.116} & $3.4(3.0-3.9)$ & \multirow{4}{*}{0.084} \\
\hline Eventual & $14.9(13.5-16.4)$ & & $3.6(3.1-4.2)$ & \\
\hline$\leq 20 \mathrm{Horas} / \mathrm{dia}$ & $15.5(14.0-17.2)$ & & $3.8(3.4-4.3)$ & \\
\hline$>20$ Horas/dia & $18.6(17.1-20.1)$ & & $5.1(4.5-5.8)$ & \\
\hline \multicolumn{5}{|l|}{ Lazer e Tempo Livre } \\
\hline Ativo Fisicamente & $11.6(10.3-13.0)$ & & $2.9(2.6-3.3)$ & \\
\hline Sedentário & $20.1(18.6-21.7)$ & 0.019 & $4.8(4.2-5.5)$ & 0.027 \\
\hline
\end{tabular}

das prevalências de sobrepeso, as dimensões das $\mathrm{OR}$ encontradas sugerem que as prevalências de sobrepeso observadas não apresentam diferenças significativas entre aqueles escolares que relataram possuir três ou mais irmãos.

No que se refere à alimentação realizada no ambiente escolar, aqueles escolares que relataram consumir alimentos comercializados nas cantinas das escolas demonstram possuir risco próximo de duas a duas vezes e meia maior de apresentar sobrepeso em comparação com seus pares que relataram não fazer uso de qualquer tipo de alimento durante o período que permanecem na escola (valores brutos $-\mathrm{OR}=$ 2,66; 95\% IC 2,25 - 3,14; valores ajustados $-\mathrm{OR}=$ 2,09; 95\% IC 1,74-2,49). Neste sentido, destaca-se que mediante ajuste pelas demais variáveis independentes do modelo de regressão logística, os escolares que se alimentam da merenda oferecida pelas escolas e os escolares que não fazem uso de qualquer tipo de alimento durante o período que permanecem na escola demonstram chances no campo estatístico similares de apresentar sobrepeso $(\mathrm{OR}=1,11 ; 95 \%$ IC 0,89 - 1,32).

A distância que os escolares relataram percorrer para chegar a escola também se define como um fator de impacto na ocorrência do excesso de massa corporal. As estimativas encontradas revelam que as prevalências associadas ao sobrepeso apresentaram valores progressivamente maiores de acordo com a maior proximidade entre a residência dos escolares e a escola que freqüentam. Os escolares que relataram residir a menos de um quilômetro das escolas que freqüentam demonstraram possuir risco, em valores aproximados, entre $67 \%$ e $35 \%$ maior de apresentarem sobrepeso (valores brutos - OR $=1,67 ; 95 \%$ IC 1,39 - 1,97; valores ajustados - $\mathrm{OR}=1,35 ; 95 \%$ IC $1,09-1,67)$ que escolares os quais relataram ser necessário se deslocar mais de $10 \mathrm{~km}$ para chegar a escola.

Quanto ao meio de transporte utilizado para ir a escola, aqueles escolares que apontaram se deslocarem utilizando automóveis, mediante ajuste para as demais variáveis consideradas no estudo, demonstraram risco $58 \%$ mais elevado de apresentar sobrepeso $(\mathrm{OR}=1,58 ; 95 \%$ IC $1,31-1,90) \mathrm{em}$ comparação com aqueles escolares que relataram se deslocarem para escola caminhando ou de bicicleta. Escolares que relataram utilizar transporte coletivo para se deslocar até a escola também apresentaram maior predisposição para a ocorrência do sobrepeso comparativamente aos escolares que relataram se deslocar caminhando ou de bicicleta $(\mathrm{OR}=1,31$; 95\% IC 1,05-1,61).

$\mathrm{O}$ fato de os escolares estarem envolvidos com algum tipo de trabalho remunerado também se caracterizou como componente de risco para a ocorrência do sobrepeso. Aqueles que relataram possuir jornada de trabalho superior a 20 horas/ semana demonstram possuir risco 37\% maior de apresentarem sobrepeso $(\mathrm{OR}=1,37$; 95\% IC 1,11 
Tabela 3. Valores de odds ratio com $95 \%$ de intervalo de confiança (IC) do sobrepeso de acordo com selecionadas variáveis sociodemográficas e comportamentais da população escolar de Montes Claros, Minas Gerais, Brasil, 2007.

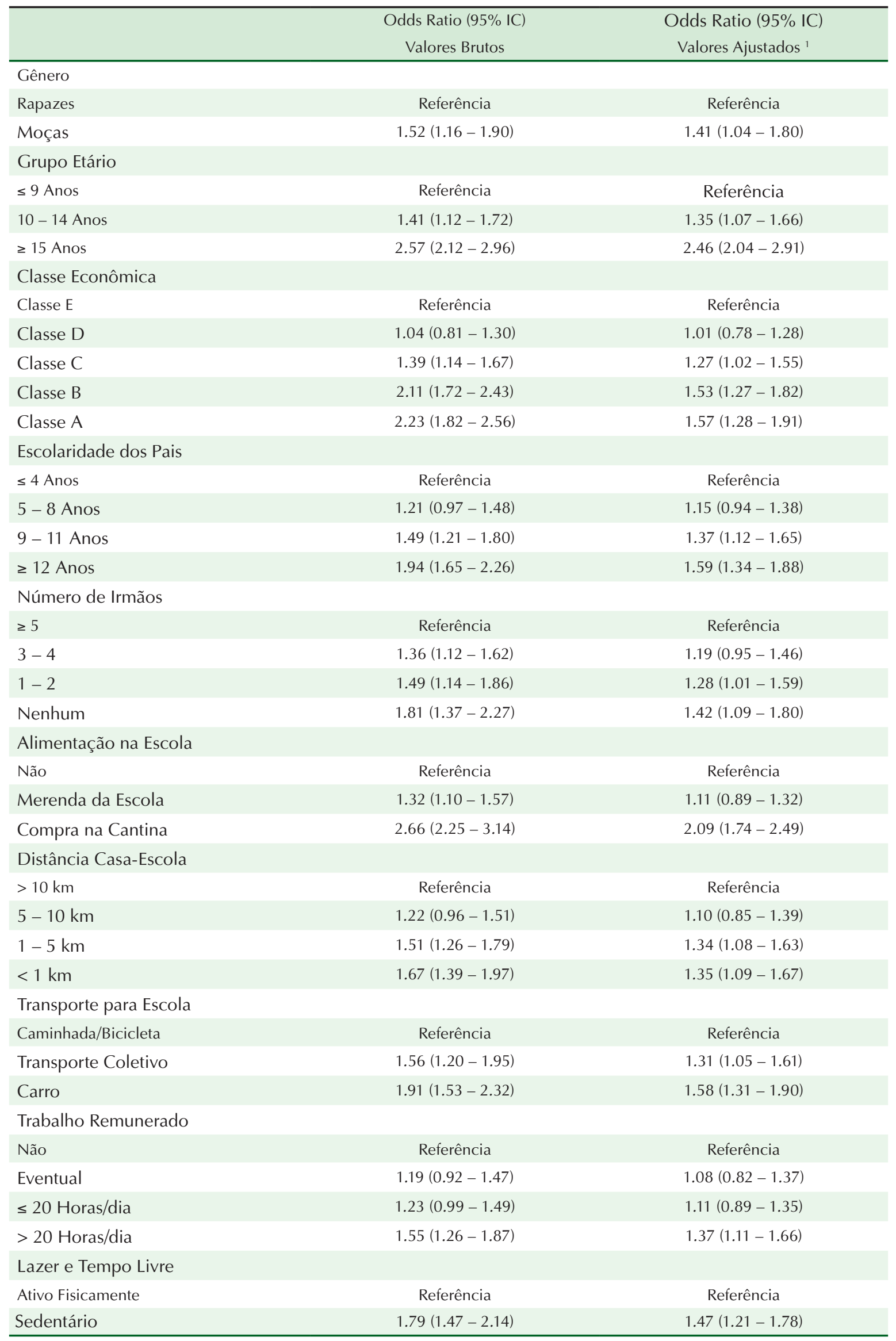

${ }^{1}$ Valores ajustados pelas demais variáveis independentes do modelo de regressão 
- 1,66) em comparação aos escolares que relataram não possuir qualquer atividade laboral, independentemente do impacto de outras variáveis tratadas no modelo de regressão.

No que se refere ao lazer e à ocupação do tempo livre, os resultados apontam que os escolares ao preferirem se ocupar com atividades sedentárias no seu lazer, demonstram significativamente maior vulnerabilidade para acumular quantidades excessivas de massa corporal que seus pares os quais preferem se envolver com um lazer ativo. Essa maior vulnerabilidade, ajustada pelas demais variáveis consideradas no estudo, alcança proporção próxima de $50 \%(\mathrm{OR}=1,47$; 95\% IC 1,21 - 1,78).

\section{DISCUSSÃO}

Os resultados encontrados revelaram que, utilizando-se dos pontos-de-corte específicos para gênero e idade propostos pela IOTF, as estimativas de prevalência de sobrepeso e obesidade na população escolar analisada foram equivalentes a 17,2\% e $3,8 \%$, respectivamente. Neste sentido, a ausência de unanimidade na definição de pontos-de-corte a serem empregados em idades jovens para identificação do sobrepeso e da obesidade se apresenta como o maior obstáculo quando da realização de análises comparativas entre diferentes estudos disponibilizados na literatura. No entanto, quando da confrontação com dados internacionais, que também empregaram os pontos-de-corte propostos pela IOFT, a prevalência de sobrepeso e obesidade observada no presente estudo foi inferior a descrita na população jovem de países da América do Norte e europeus ${ }^{1}$; porém, superior as prevalências identificadas recentemente na população jovem de países asiáticos ${ }^{9,10}$ e africanos ${ }^{11,12}$.

Por outro lado, limitação importante freqüentemente observada em estudos nacionais e que dificulta comparações entre as prevalências de sobrepeso e obesidade de jovens de diferentes regiões brasileiras, refere-se aos delineamentos metodológicos utilizados, via de regra, selecionando amostras não representativas do segmento populacional estudado. Ainda, raros são os estudos nacionais que procuraram analisar amostras de crianças e adolescentes conjuntamente. Além disso, os agrupamentos dos dados por faixas etárias não são consensuais. Por esses motivos, optou-se por estabelecer comparações unicamente com dados da Pesquisa de Orçamentos Familiares (POF), coletados em 2002 - 2003, envolvendo amostras de jovens entre 10 e 19 anos de todo o território brasileiro².
$\mathrm{Na}$ POF o sobrepeso foi definido mediante valores equivalentes ao IMC para gênero e idade iguais ou superiores ao percentil 85 e foram considerados como obesos aqueles jovens que apresentavam valores equivalentes ao IMC para gênero e idade iguais ou superiores ao percentil $95 . \mathrm{Na}$ pesquisa foram utilizadas as referências normativas construídas com base em estudos norte-americanos. Com relação à prevalência de sobrepeso, a proporção encontrada no presente estudo foi bastante similar à encontrada na $\mathrm{POF}(17,2 \%$ versus $16,7 \%)$. No entanto, a prevalência de obesidade encontrada na população escolar aqui analisada foi superior à encontrada na POF (3,8\% versus 2,3\%). Chamase atenção para o fato de estudos nacionais terem atestado que os valores equivalentes ao IMC da população jovem norte-americana correspondente aos percentis 85 e 95, utilizados como ponto-de-corte na $\mathrm{POF}$, são mais elevados que os valores equivalentes ao IMC nos mesmos percentis observados em estudos anteriores na população brasileira ${ }^{13}$, o que pode ter subestimado a real proporção de prevalência de sobrepeso e obesidade dos jovens brasileiros analisados pela POF. As diferenças quanto aos agrupamentos etários considerados no presente estudo e no levantamento nacional é mais um fator limitante que deverá ser levado em conta nas comparações realizadas.

As prevalências de sobrepeso não se distribuíram igualmente na população escolar analisada no presente estudo. Gênero, idade e selecionados fatores sociodemográficos e comportamentais demonstraram participações distintas na distribuição e no risco de os escolares analisados apresentarem sobrepeso. Quanto ao gênero, as moças apresentaram prevalências de sobrepeso mais elevadas que os rapazes. Em outros estudos disponibilizados na literatura, também foram encontradas importantes diferenças quanto às prevalências de sobrepeso entre ambos os gêneros, não sendo apresentadas justificativas bem definidas para esta ocorrência ${ }^{1,}$ 9-12. Suspeita-se que as mais elevadas prevalências entre as moças possam ser parcialmente explicadas pela maior vulnerabilidade do organismo feminino, ocasionada pelos hormônios sexuais em idades próximas à puberdade e ao aumento dos estoques de gordura corporal ${ }^{14}$. Diferenças quanto aos hábitos de prática de atividade física também podem contribuir para as variações nas prevalências de sobrepeso e obesidade entre ambos os gêneros. Em idades escolares, os rapazes demonstram ser consistentemente mais ativos fisicamente que as moças, diferenças que se acentuam com a adolescência ${ }^{15}$. 
Corroborando informações apresentadas por outros estudos ${ }^{16,17}$, nossos resultados também apontaram para o fato de o sobrepeso ser mais prevalente entre os jovens de classes econômicas mais favorecidas. Contudo, preocupa o fato de elevada proporção de escolares posicionados em classes econômicas mais baixas também apresentarem sobrepeso e obesidade. Tradicionalmente, o excesso de massa corporal era sinal de abundância e riqueza; logo, fenômeno típico de classes econômicas mais favorecidas. Porém, atualmente, elevadas prevalências de sobrepeso estão sendo identificadas também em classes econômicas e em níveis de escolarização mais baixos ${ }^{18}$.

Em tese, o ganho excessivo de massa corporal é dependente do equilíbrio energético positivo, estabelecido quando o consumo excede o dispêndio de energia em interação com a suscetibilidade genética. Ainda, entre os jovens, o ato de se alimentar, apesar de fisiológico, é condicionado pela disponibilidade de recursos financeiros para a aquisição dos alimentos e pela educação familiar para a escolha e a preparação desses alimentos. Neste caso, pressupõe-se que os escolares pertencentes às famílias de classe econômica menos favorecidas, em razão de seu menor poder aquisitivo e de sua maior fragilidade no campo da educação alimentar, estariam mais expostos à influência da mídia, que incentiva o uso de produtos de elevada densidade calórica, ricos em gorduras e carboidratos simples, porém de menor custo e de mais fácil acesso ${ }^{19}$. Também, há de se considerar as mudanças observadas mais recentemente no estilo de vida dos jovens, independentemente da classe econômica a que pertencem: mais tempo frente à televisão, substituição de brincadeiras mais intensas fisicamente por jogos sedentários, dificuldade de brincar na rua em razão da falta de segurança, os quais têm reduzido drasticamente o dispêndio energético ${ }^{15}$.

Outro fator que demonstrou significativo impacto para a ocorrência do sobrepeso foi o mais elevado nível de escolarização dos pais ou dos responsáveis pelos escolares analisados no estudo. Esses achados não confirmam evidências encontradas por alguns estudos disponibilizados na literatura que apontam relação inversa entre os níveis de escolarização dos pais e a presença do sobrepeso nos filhos ${ }^{20}$. Neste aspecto, a princípio, pode-se assumir que o nível de escolarização oferece fortes indicações quanto à condição econômica e ao status educacional da família, o que influencia, entre outros comportamentos do convívio familiar, nas escolhas dos alimentos e nas opções de prática da atividade física dos filhos. Vale salientar que, no delineamento empregado no estudo a classe econômica foi uma das variáveis controladas estatisticamente. Sendo assim, os resultados aqui encontrados apontam que a associação entre o nível de escolarização dos pais e a ocorrência de sobrepeso nos filhos possa depender do contexto cultural e educacional em que a família esta inserida.

Com relação às variáveis associadas ao núcleo familiar, os resultados encontrados apontaram que escolares filhos únicos ou que têm até dois irmãos aumentaram significativamente a probabilidade de apresentar sobrepeso. O possível impacto do número de irmãos na ocorrência de sobrepeso e obesidade também tem sido analisado mediante estudos envolvendo jovens europeus ${ }^{21}$ e norte-americanos ${ }^{22}$. Nesses estudos também se observou que o risco de ocorrência de sobrepeso é inversamente proporcional ao número de irmãos, independentemente da ordem de nascimento. Neste caso, aventa-se a possibilidade de um aumento no consumo alimentar per capita, em função da diminuição do número de moradores, poderia facilitar o aparecimento do sobrepeso.

Quanto ao ambiente escolar, algumas variáveis chamam a atenção: tipo de alimentação consumida na escola, meio de transporte utilizado e distância entre a residência e a escola. Neste caso, pode-se tentar justificar a maior prevalência de sobrepeso entre os escolares que relataram consumir alimentos comercializados nas cantinas das escolas, em comparação com seus pares que não fazem uso de qualquer tipo de alimento durante o período que permanecem na escola, em razão da grande quantidade de produtos comercializados nas cantinas escolares serem de elevada densidade calórica, ricos em gorduras e carboidratos simples, o que favorece o aumento da massa corporal dos escolares que optam por esta prática.

Com relação ao meio de transporte utilizado para se deslocar até a escola e a distância entre a residência e a escola, verificou-se que os jovens conduzidos por meio de veículos automotores ou que residem a menos de $5 \mathrm{~km}$ da escola se mostraram mais susceptíveis ao excesso de massa corporal quando comparados àqueles que se deslocavam caminhando ou de bicicleta e residiam mais distante da escola. Resultados similares foram encontrados em outro estudo envolvendo casuística selecionada na região sul do Brasil, apontando que escolares com menores valores equivalentes ao IMC optavam por se deslocar ativamente até a escola ${ }^{23}$. Iniciativas com intuito de minimizar o risco direcionado ao aumento da massa corporal dos escolares deverão contemplar estratégias de intervenção que estimu- 
lem os escolares a se deslocar para escola de forma ativa. Neste caso, alguns aspectos de urbanização podem influenciar para que os escolares venham a se deslocar ativamente às escolas, como é o caso da pavimentação dos acessos, transito organizado, segurança e, sobretudo, informação quanto a necessidade de se adotar um estilo de vida ativo fisicamente desde as idades escolares.

O envolvimento em trabalho remunerado foi outro fator de impacto identificado que contribuiu para a maior ocorrência do sobrepeso entre os escolares amostrados. Os resultados encontrados sugerem que escolares com jornada de trabalho $>20$ horas/dia demonstraram risco significativamente mais elevado de apresentar sobrepeso em comparação com seus pares que relataram não realizar trabalho remunerado. A justificativa para que a jornada de trabalho $>20$ horas/dia possa estar positivamente associada ao sobrepeso dos escolares pode fundamentar-se no pressuposto de que, recebendo remuneração pode apresentar alguma independência financeira para a aquisição de maior quantidade de alimentos industrializados e de elevada concentração de gorduras a sua escolha, a qual repercuti na ocorrência do sobrepeso. Também, muito da possível participação dos jovens em atividade física mais intensa necessariamente deva ser realizada fora do ambiente escolar. Contudo, para que isso venha acontecer, os jovens necessitam de disponibilidade de tempo que lhes permita aderir a essas atividades que normalmente decorrem em organizações esportivas (clubes, associações, ginásios), o que entre escolares trabalhadores diminui esta possibilidade em razão do trabalho remunerado ocupar porção significativa desse tempo.

No presente estudo foi encontrado, ainda, forte impacto das atividades sedentárias realizadas no lazer e na ocupação do tempo livre na ocorrência do sobrepeso. Outros estudos também descreveram a associação positiva existente entre o sedentarismo e o aumento da massa corporal na população jovem 1, 15. O comportamento sedentário e o sobrepeso podem resultar em um aumento no aparecimento e no desenvolvimento de morbidades na idade jovem com grande repercussão na idade adulta ${ }^{24,25}$.

\section{CONCLUSÃO}

Os achados do presente estudo mostram que, embora as prevalências de sobrepeso e obesidade sejam comparáveis com estimativas observadas em outras regiões brasileiras e menores que as encontradas em regiões mais industrializadas, a proporção de escolares que apresentam sobrepeso e são obesos é particularmente preocupante. Nesta população de escolares os principais fatores sociodemográficos e comportamentais identificados na ocorrência do sobrepeso e da obesidade foram classe econômica familiar, escolaridade dos pais, número de irmãos, meio de transporte e distância entre residência e escola, tipo de alimentação consumida na escola, executar trabalho remunerado, atividades de lazer e ocupação do tempo livre.

Informações acumuladas em estudos epidemiológicos revelam que as prevalências de sobrepeso e obesidade são maiores em países desenvolvidos quando comparadas com país em desenvolvimento, relação que apresenta chance de modificação somente pela cultura da população e pelo acesso a informações vinculadas ao controle da massa corporal e aos serviços de saúde. Dessa maneira, na tentativa de explicar os resultados encontrados quanto ao comportamento das prevalências de sobrepeso e obesidade no presente estudo, apresentase a hipótese de que, os jovens aqui analisados, pertencentes a uma comunidade ainda em processo de desenvolvimento, poderiam estar importando hábitos alimentares e de prática de atividade física de países desenvolvidos sem, no entanto, terem acesso a informações e a políticas de saúde pública que possam oferecer eventual proteção ao excessivo acúmulo de massa corporal. Destaca-se que fenômeno idêntico possa estar ocorrendo em outras comunidades da sociedade brasileira, o que contribui para a epidemia de sobrepeso e obesidade que está sendo formada na América Latina.

Diante da relação inversa entre o maior acúmulo de massa corporal e o melhor estado de saúde, e pela acentuada estabilidade longitudinal do sobrepeso e da obesidade entre jovens e adultos, os resultados aqui encontrados sugerem a necessidade de implementar programas intervencionistas sistematizados na população analisada. Neste sentido, destaca-se que os programas direcionados à prevenção, ao combate e ao controle do sobrepeso e da obesidade, necessariamente, deverão combinar ações quanto à restrição moderada do suprimento energético, à oferta de distribuição adequada de macro e micro nutrientes e à prática regular de exercícios físicos específicos que venha a elevar o dispêndio energético.

\section{REFERÊNCIAS BIBLIOGRÁFICAS}

1. Janssen I, Katzmarzyk PT, Boyce WF, Verrecken C, Mulvihill C, Roberts Cet al. Comparison of overweight and obesity prevalence in school-aged youth from 34 
countries and their relationships with physical activity and dietary patterns. Obes Rev 2005;6:123-32.

2. Instituto Brasileiro de Geografia e estatística - IBGE. Pesquisa de Orçamentos Familiares - Antropometria e Análise do Estado Nutricional de Crianças e Adolescentes no Brasil. Rio de Janeiro: IBGE. 2006.

3. World Health Organization - WHO. Obesity: Preventing and Managing the Global Epidemic. Report of a WHO Consultation on Obesity. Geneva: World Health Organization, 2000.

4. Kiess W, Galler A, Reich A, Muller G, Kapellen T, Deutscher J, Raile K, Kratzsch J. Clinical aspects of obesity in children and adolescence. Obes Rev 2001;2:29-36.

5. Programa das Nações Unidas para o Desenvolvimento - PNUD. Relatório de Desenvolvimento Humano 2007/2008. Brasília: UNUD. 2007.

6. Associação Brasileira de Institutos de Pesquisa de Mercado - ABIPEME. Critério de Classificação Econômica Brasil. São Paulo. 2007.

7. Cole TJ, Bellizzi MC, Flegal KM, Dietz WH. Establishing a standard definition for child overweight and obesity worldwide: international survey. BMJ 2000; 320:1240-3.

8. World Health Organization (WHO). Physical Status: The Use and Interpretation of Anthropometry. Report of a WHO Expert Committee. WHO - Technical Report Series, v.854, p.1-452, 1995.

9. Laxmaiah A, Nagalla B, Vijayaraghavan K, Nair M. Factors affeting prevalence on overweight among 12 to 17-year-old urban adolescents in Hyderabad-Índia. Obesity (Silver Spring) 2007;15:1384-90.

10. Xie B, Chou CP, Spruijt-Metz D, Reynolds K, Clark F, Palmer PH, Gallaher P, Sun P, Guo Q, Johnson CA. Socio-demographic and economic correlates of overweight status in Chinese adolescents. Am J Health Behav 2007;31:339-52.

11. Ben-Bassey UP, Oduwole AO, Ogundipe OO. Prevalence of overweight and obesity in Eti-Osa LGA, Lagos, Nigeria. Obesity Review 2007;8:475-9.

12. Armstrong ME, Lambert MI, Sharwood KA, Lambert EV. Obesity and overweight in South African primary school children - The Health of the Nation Study. S Afr Med J 2006;96:439-44.

13. Veiga GV, Dias PC, Anjos LA. A comparison of distribution curves of body mass index from Brazil and United States for assessing overweight and obesity in Brazilian adolescents. Rev Panam Salud Pub 2001;10:79-85.

14. Malina RM, Bouchard C, Bar-Or O. Growth, Maturation, and Physical Activity. $2^{\text {nd }}$ Edition. Champaign, Illinois: Human Kinetics. 2004.
15. Ferreira I, Van der Horst K, Wendel-Vos W, Kremers S, Van Lenthe FJ, Brug J. Environmental correlates of physical activity in youth: a review and update. Obes Rev 2006;8:129-54.

16. Neutzling MB, Taddei JAAC, Rodrigues EM, Sigulem DM. Overweight and obesity in Brazilian adolescents. Int J Obes 2000;24:869-74.

17. Troiano RP, Flegal KM. Overweight children and adolescents: description, epidemiology, and demographics. Pediatrics 1998;101:497-504

18. McLellan F. Obesity rising to alarming levels around the world. Lancet 2002;359:1412.

19. Drewnowsk A, Popkin BM. The nutrition transition: new trends in the global diet. Nutrition Review 1997;55:31-43.

20. Gnavi R, Spagnoli TD, Galotto C, Pugliese E, Carta A, Cesan L. Socioeconomic status, overweight and obesity in prepuberal children: a study in an area of Northern Italy. Eur J Epidemiol 2000;16:797-803.

21. Ravalli GP, Belmont L. Obesity in nineteen-year-old men: family size and birth order associations. Am J Epidemiol 1979;109:66-70.

22. Stettler N, Tershakovec AM, Zemel DS, Leonard MB, Boston RC, Katz, SH. Early risk factors for increased adiposity: a cohort study of African American subjects followed from birth to young adulthood. Am J Clin Nutr 2000;72:378-83.

23. Hallal PC, Bertoldi AD, Gonçalves H, Victora CG. Prevalência de sedentarismo e fatores associados em adolescentes de 10-12 anos de idade. Cad Saúde Pública 2006;22:1277-87.

24. Ori F, Palomba S, Cascella T, Savastano S, Lombardi G, Colao G. Cardiovascular complications of obesity in adolescents. J Endocrinol Envest 2007;30:70-80.

25. Ryu SY, Kweon SS, Park HC, Shin JH, Rhee JA. Obesity and the metabolic syndrome in Korean adolescents. J Korean Med Sci 2007;22:513-7.
Endereço para correspondência
Dartagnan Pinto Guedes
Rua Ildefonso Werner 177
Condomínio Royal Golf
CEP 86055-545 - Londrina, Paraná, Brasil
E-mail: darta@sercomtel.com.br 